\title{
Estimando a magnitude do parâmetro de looping do meandro do vento horizontal
}

Obtaining the meandering loop parameter in low wind speed condition

\author{
Lilian P. Moor ${ }^{1,3}$, Gervasio A. Degrazia ${ }^{2}$, Michel B. Stefanello ${ }^{2}$, Luca Mortarini ${ }^{4}$, Otavio C. \\ Acevedo $^{2}$, Silvana Maldaner ${ }^{5}$, Charles R. P. Szinvelski ${ }^{6}$, Débora R. Roberti' ${ }^{2}$, Lidiane Buligon ${ }^{6}$ e \\ Domenico Anfossi ${ }^{4}$
}
${ }^{1}$ Professora da Coordenação Geral de Ensino, Instituto Federal Farroupilha, Jaguari, RS, Aluno(a) da PPGFis Universidade Federal de Santa Maria, Santa Maria, RS, Brasil lilimoor@gmail.com
${ }^{2}$ Professor(a) do Departamento de Física, Universidade Federal de Santa Maria, Santa Maria, RS, Brasil gervasiodegrazia@gmail.com; michelstefanello@gmail.com; otavio@ufsm.br; debora@ufsm.br
${ }^{3}$ Professora da Coordenação Geral de Ensino, Instituto Federal Farroupilha, Jaguari, RS, Brasil
${ }^{4}$ Pesquisador do Institute of Atmospheric Sciences and Climate (ISAC) of the Italian National Research Council (CNR), Unit of Turin, Italy
1.mortarini@isac.cnr.it; d.anfossi@isac.cnr.it
${ }^{5}$ Professora da Coordenadoria Acadêmica, Universidade Federal de Santa Maria-campus Cachoeira do Sul, RS, Brasil
silvana.maldaner@gmail.com
${ }^{6}$ Professor(a) do Departamento de Matemática, Universidade Federal de Santa Maria, Santa Maria, RS, Brasi charlessz45@gmail.com; prof.buligon@gmail.com

\section{Resumo}

A magnitude do parâmetro de looping $m$ é o principal critério utilizado para caracterizar o meandro do vento do horizontal. Es te parâmet está associado à magnitude do lóbulo negativo da função de autocorrelação experimental das componentes horizontais da velocidade. Nes trabalho apresenta-se um estudo dos valores médios do parâmetro de looping $m$ do meandro do vento horizontal utilizando dados de 2 síti situados em diferentes latitudes.

Palavras-chave: meandro do vento, função de autocorrelação.

\begin{abstract}
The looping parameter $m$ is the main value to characterize the meandering phenomenon. This parameter is relationship with negative lob in the observed autocorrelation function generated from components of horizontal speed. In this work, we present a study comparing ti mean values of the looping parameter between 2 diverse sites in the Brazilian sector.

Keywords: Meandering, autocorrelation function.
\end{abstract}




\section{Introdução}

O fenômeno de meandro está associado às grandes oscilações direcionais do vento médio horizontal e surge quando a magnitude da velocidade é muito baixa (menor que $1.5 \mathrm{~ms}^{-1}$ ) (Anfossi et al., 2005). Estados físicos associados à condição de baixa velocidade do vento são caracterizados pela coexistência de turbulência e meandro (Goulart et al., 2007). Estes escoamentos complexos são difíceis de serem descritos. Nos últimos anos, diversos trabalhos foram dedicados ao estudo do meandro do vento horizontal. Estes resultados permitiram um entendimento mais profundo acerca do fenômeno (Anfossi et al., 2005; Goulart et al., 2007; Mortarini et al., 2013). As últimas investigações mostraram que o meandro pode ocorrer sob qualquer relevo, latitude ou horário do dia. Um marcador de meandro é o lóbulo negativo presente na função de autocorrelação (FA) experimental gerada a partir das componentes horizontais da velocidade do vento. As FAs fornecem parâmetros importantes para a caracterização do meandro, como o período de meandro $\left(T_{*}\right)$, que assume valores médios de 2000 segundos (Anfossi et al., 2005; Moor et al., 2015) e o parâmetro de looping $m$, que está associado a intensidade do lóbulo negativo da FA. Este parâmetro é o principal diferenciador entre a presença de uma turbulência bem desenvolvida e o meandro. Grandes valores de $m(m>>1)$ indicam a ocorrência de meandro. Quando este valor se aproxima de zero, o fenômeno de meandro desaparece e a turbulência passa a dominar o escoamento (Degrazia et al., 2008).

Neste trabalho apresenta-se um estudo comparativo do valor médio do parâmetro de looping $m$, entre 2 diferentes sítios geográficos, localizados no Brasil.

\section{Metodologia e Resultados}

Para o desenvolvimento deste trabalho foram utilizados observações medidas em 2 sítios situados em diferentes latitudes no território brasileiro. Um conjunto de dados coletado em uma região de pastagem, situada ao longo da rodovia que interliga Santarém e Cuiabá, no km77, no estado do Pará, com as seguintes coordenadas: latitude de $-3.0118955^{\circ}$ e longitude de $-54.53652^{\circ}$ (fluxnet.ornl.gov, 2013). Estes dados fazem parte do projeto LBA-ECO (Large Scale Biosphere-Atmosphere Experiment in Amazon) que teve duração de vários anos $\mathrm{e}$ contou com a participação de diversos países (para este estudo foi utilizado o período de janeiro-outubro/2003). As medidas foram registradas por um anemômetro sônico 3D (tipo SATI/3K Applied Technologies, Inc., Longmont, CO, USA) com uma frequência amostral de 10 Hz. O equipamento estava instalado a uma altura de 8,75 $\mathrm{m}$ acima do solo e direcionado para leste, direção preferencial do vento na região (Acevedo et al., 2004). O segundo conjunto de dados foi coletado na Universidade Federal de Santa Maria-UFSM, com as seguintes coordenadas: latitude de $-53.7597^{\circ}$ e longitude de $-29.7241^{\circ}$. O anemômetro sônico está instalado a $3 \mathrm{~m}$ acima do solo em uma área plana que faz parte do bioma Pampa. A frequência amostral é de $10 \mathrm{~Hz}$. Foram usados os dados referentes ao período de novembro/2013 a dezembro/2014.

Foram analisadas séries temporais de 1 hora para o período noturno. O conjunto de dados da Amazônia gerou um total de 1474 casos com a presença de meandro e Santa Maria gerou um total de 1295 casos. Cada série temporal resultou em uma FA experimental (indicador da existência de meandro). Para ajustar os dados experimentais foi utilizada a nova formulação para a FA que descreve o fenômeno do meandro, descrita por (Moor et al., 2015)

$$
R_{u, v}(\tau)=\frac{\cos \left(q_{u, v} \tau\right)}{\left(1+p_{u, v} \tau\right)^{2}}
$$

onde $p_{u, v}$ e $q_{u, v}$ são quantidades híbridas expressas em termos da escala de tempo integral $\left(T_{u, v}\right)$ e do parâmetro de looping $m$. Estas quantidades são extraídas dos ajustes das FAs observadas com a Eq. (1) e também podem ser fornecidas por (Mortarini et al., 2013)

$$
p_{u, v}=\frac{1}{\left(m_{u, v}^{2}+1\right) T_{u, v}}
$$

$\mathrm{e}$

$$
q_{u, v}=\frac{m_{u, v}}{\left(m_{u, v}^{2}+1\right) T_{u, v}}
$$


O parâmetro $m$ é um parâmetro adimensional que controla a frequência de oscilação do meandro, e se torna muito importante quando estudamos condições associadas à calma de vento. Quanto maior o valor do $\mathrm{m}$ maior será o lóbulo negativo gerado pela FA. As Fig. 1 e 2 mostram FAs observadas na Amazônia brasileira e ajustadas com a Eq. (1). A Fig. 1 tem um valor de $m=7$ menor que a Fig. $2(m=8)$. Os estudos apontam que quanto maior a magnitude de $\mathrm{m}$ maior é a intensidade do fenômeno meando.

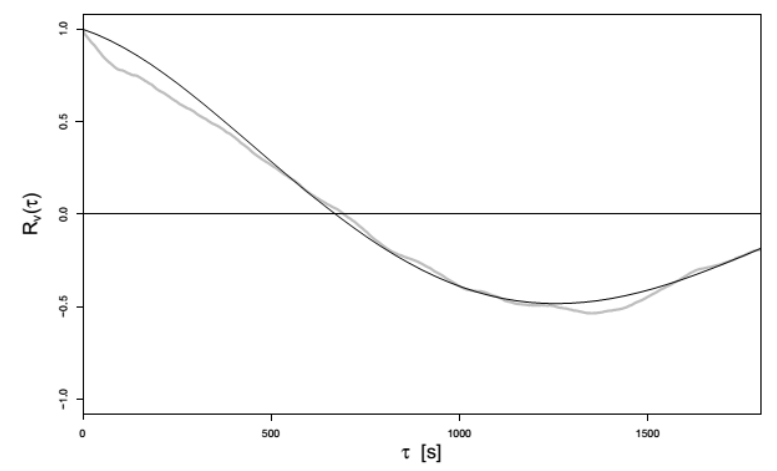

Figura 1 - FA da componente $\mathrm{u}$ do vento às $22 \mathrm{~h}$ do dia 9 de janeiro de 2003 (Amazônia).

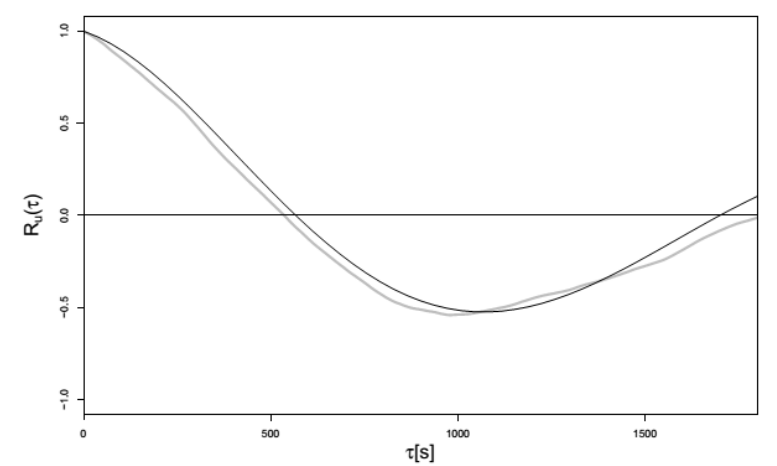

Figura 2 - FA da componente $u$ do vento para à 01h do dia 08 de janeiro de 2003 (Amazônia).

Buligon (2013) derivou uma expressão para a taxa de dissipação $\varepsilon$ introduzindo a Eq. (1) no modelo de Taylor e obteve a seguinte expressão

$$
\varepsilon=4 p \frac{4}{\left(1+m^{2}\right)} \frac{\sigma_{v}^{2}}{C_{0} T_{L}}
$$

assim

(i) $m=0 \Rightarrow \varepsilon=4 \frac{\sigma_{v}^{2}}{C_{0} T_{L}}$ (ii) $m=1 \Rightarrow \varepsilon=2 \frac{\sigma_{v}^{2}}{C_{0} T_{L}}$

(iii) $m=\infty \Rightarrow \varepsilon \rightarrow 0$

Dessa forma pode-se verificar que à medida que $m$ aumenta e o fenômeno de meandro começa a se manifestar, a taxa de dissipação turbulenta diminui. Este comportamento é fisicamente razoável, uma vez que as oscilações de baixa frequência são relacionadas a números de onda muito menores do que aqueles associados à dissipação molecular.

Para determinar o valor do parâmetro de looping $m$ utilizamos a seguinte relação

$$
m=q / p
$$

Os valores médios obtidos para $m$ para este estudo estão apresentados na Tabela 1.

Tabela 1 - Valores médios para $m$.

\begin{tabular}{c|c|c}
\hline & Amazônia & Santa Maria \\
\hline $\bar{u}$ & $0.45 \mathrm{~ms}^{-1}$ & $0.50 \mathrm{~ms}^{-1}$ \\
\hline$m_{u}$ & 4.7 & 3.8 \\
\hline$m_{v}$ & 5.1 & 3.3 \\
\hline
\end{tabular}

Neste estudo também se observou uma maior ocorrência do meandro na Amazônia, que representou $80 \%$ dos casos noturnos. Em Santa Maria foram registrados apenas $40 \%$ de casos de meandro.

\section{Conclusões}

O presente trabalho apresenta uma comparação dos valores do parâmetro de looping $m$ em 2 sítios. O resultado da Tabela 1 mostra que os valores encontrados para a Amazônia apresentam maior magnitude do que os valores encontrados na região de Santa Maria. Estas magnitudes de $m$ podem ser utilizadas em modelos de dispersão de contaminantes descrevendo a difusão reforçada provocada pelo meandro. 


\section{Agradecimentos}

Os autores gostariam de agradecer à Coordenação de Aperfeiçoamento de Pessoal de Nível Superior (CAPES) pelo suporte financeiro.

\section{Referências}

Acevedo, O. C., Moraes, O. L., Da Silva, R., Fitzjarrald, D. R., Sakai, R. K., Staebler, R. M., \& Czikowsky, M. J. (2004). Inferring nocturnal surface fluxes from vertical profiles of scalars in an Amazon pasture. Global Change Biology, 10, 1-9.

Anfossi, D.; Degrazia, G. A.; Goulart, A. (2005). An Analysis of Sonic Anemometer Observations in Low Wind Speed Conditions. Boundary-Layer Meteorology, 114, 179-203.

Buligon, L.; Degrazia, G. A.; Szinvelski, C.; Moor, L. P. (2013). Uma nova derivação da taxa de dissipação turbulenta para eventos de turbulência fraca e bem desenvolvida. Ciência e Natura, v. X, p. 270.

Degrazia, G. A.; A. Goulart; J. Costa Carvalho3, C. R. P. Szinvelski; L. Buligon and A. Ucker Timm. (2008). Turbulence dissipation rate derivation for meandering occurrences in a stable planetary boundary layer. Atmos. Chem. Phys., 8. 1713-1721.

Fluxnet.ornl.gov. Fluxnet, 2013. Disponível em: $<$ http://fluxnet.ornl.gov/site/84>. Acesso em: 01 dez. 2014.

Goulart A.; Degrazia G.; Acevedo O.; Anfossi D. (2007). Theoretical considerations of meandering winds in simplified conditions. Bound.-Lay. Meteorol., 125, 279-287.

Moor, L. P., Gervasio A. Degrazia; Michel B. Stefanello; Luca Mortarini; Otavio C. Acevedo; Silvana Maldaner; Charles R. P. Szinvelski; Débora R. Roberti; Lidiane Buligon; Domenico Anfossi. (2015). Proposal of a new autocorrelation function in low wind speed conditions. PhysicaA: Statistical Mechanics and its Applications, vol. 438, p. 286 - 292.

Mortarini, L.; E. Ferrero; S. Falabino; S. Trini Castelli; R. Richiardone and D. Anfossi. (2013). Low-frequency processes and turbulence structure in a perturbed boundary layer. Q.J.R. Meteorol. Soc., 139, 1059-1072. 